\title{
ASO Author Reflections: A Population-Based Study on Lymph Node Retrieval in Patients with Esophageal Cancer
}

\author{
L. R. van der Werf, MD and B. P. L. Wijnhoven, MD, PhD \\ Department of Surgery, Erasmus University Medical Centre, Rotterdam, The Netherlands
}

PAST

Oesophagectomy is the cornerstone of treatment for esophageal cancer. Extended lymphadenectomy may reduce locoregional failure and improve long-term survival. However, the value of lymph node dissection for locoregional tumor control has been debated since the introduction of neoadjuvant chemoradiotherapy. Neoadjuvant chemoradiotherapy leads to tumor shrinkage and results in a higher proportion of resections with tumornegative resection margins and tumor-free lymph nodes. ${ }^{1}$

Studies have shown that the number of removed lymph nodes is associated with improved survival for patients who underwent surgery without neoadjuvant chemoradiotherapy. However, this positive correlation is lost in patients who underwent neoadjuvant chemoradiotherapy followed by surgery. ${ }^{2,3}$

Besides its role in locoregional tumor control, an extensive lymphadenectomy may improve tumor staging. Accurate pathological staging is essential for prognostication. $^{4}$

ASO Author Reflections is a brief invited commentary on the article, "A Population-based Study on Lymph Node Retrieval in Patients with Esophageal Cancer: Results from the Dutch Upper Gastrointestinal Cancer Audit," Ann Surg Oncol. 2018;25:1211-20.

(C) The Author(s) 2018

First Received: 22 September 2018; Published Online: 8 October 2018

L. R. van der Werf, MD

e-mail: L.R.vdwerf@gmail.com

\section{PRESENT}

In 2013, the number of lymph nodes removed was introduced as a quality indicator in the Dutch Upper gastrointestinal Cancer Audit (DUCA). This indicator is defined as "the percentage of patients with at least 15 retrieved LNs." The purpose of our study was to evaluate trends in the number of retrieved lymph nodes and the proportion of patients with $\geq 15$ LNs in the resection specimen.

Between 2011 and 2016, the median number of lymph nodes removed increased from 15 to 20 . The percentage of patients with at least 15 lymph nodes removed also increased from 50 to $80 \%$ on a national level. ${ }^{5}$ There was a wide variation in the percentage of patients with at least 15 lymph nodes removed between hospitals (0-77\%). In 2016, hospital variation decreased but was still considerable (between 50 and 98\%).

\section{FUTURE}

Given the importance of lymph node retrieval for locoregional control and accurate staging, a higher number of lymph nodes removed will likely improve the quality of care. However, it remains unknown if the increased number of removed lymph nodes was due to extended surgery or more accurate pathological examination of the resection specimen. The DUCA does not contain long-term followup data. Hence, the study could not report on the association of number of lymph nodes removed and survival after neoadjuvant chemoradiotherapy.

An argument for the use of this indicator in clinical auditing is that there is a clinically relevant variation in outcomes between hospitals. This may lead to improvement initiatives in the hospitals underperforming. Continues use of this indicator in the DUCA may reduce variation and improve outcomes on a national level. 
DISCLOSURE The authors have no conflicts of interest to disclose.

OPEN ACCESS This article is distributed under the terms of the Creative Commons Attribution 4.0 International License (http://crea tivecommons.org/licenses/by/4.0/), which permits unrestricted use, distribution, and reproduction in any medium, provided you give appropriate credit to the original author(s) and the source, provide a link to the Creative Commons license, and indicate if changes were made.

\section{REFERENCES}

1. van Hagen P, Hulshof MC, van Lanschot JJ, et al. Preoperative chemoradiotherapy for esophageal or junctional cancer. $N$ Engl $J$ Med. 2012;366:2074-84.
2. Talsma AK, Shapiro J, Looman CW, et al. Lymph node retrieval during esophagectomy with and without neoadjuvant chemoradiotherapy: prognostic and therapeutic impact on survival. Ann Surg. 2014;260:786-92; discussion 792-3.

3. Markar SR, Noordman BJ, Mackenzie H, et al. Multimodality treatment for esophageal adenocarcinoma: multi-center propensity-score matched study. Ann Oncol. 2017;28:519-27.

4. Shapiro J, Biermann K, van Klaveren D, et al. Prognostic value of pretreatment pathological tumor extent in patients treated with neoadjuvant chemoradiotherapy plus surgery for esophageal or junctional cancer. Ann Surg. 2017;265:356-62.

5. van der Werf LR, Dikken JL, van Berge Henegouwen MI, et al. A population-based study on lymph node retrieval in patients with esophageal cancer: results from the Dutch Upper Gastrointestinal Cancer Audit. Ann Surg Oncol. 2018;25:1211-20. 